\title{
La suplementación con Lactobacillus reuteri fue eficaz y costo ahorativa para prevenir diarreas e infecciones respiratorias en preescolares
}

\section{Lactobacillus reuteri supplementation was effective and cost-saving to prevent diarrhea and respiratory infections in preschool children}

\section{Objetivos}

Determinar si la administración diaria de Lactobacillus reuteri (LBR) DSM 17938 reduce la frecuencia y la duración de los episodios de diarrea en niños que concurren a guarderías en México.

\section{Diseño, lugar y participantes}

Ensayo clínico prospectivo, aleatorizado, doble ciego, controlado y comparado con placebo realizado entre abril de 2011 y junio de 2012, y con un seguimiento de 12 semanas. Fueron incluidos niños de ambos sexos, de 6 a 36 meses de edad, nacidos de término, de similar clase social, atendidos en cuatro guarderías del sudeste de México DF.

Criterios de exclusión: bajo peso al nacer, enfermedades crónicas, alergias, retraso del crecimiento, y exposición a antibióticos, prebióticos y probióticos durante las cuatro semanas previas al estudio.

El resultado principal evaluado fue el número de días con diarrea por niño (tres o más deposiciones líquidas en 24 horas). Fueron resultados secundarios los días con infecciones de las vías aéreas, el ausentismo escolar, el uso de antibióticos, el número de consultas médicas y los costos. Se realizó un análi-
Gutierrez-Castrellon P y col. Pediatrics 2014, 133 (4): 904-9. sis de costo-efectividad comparando los resultados en salud y los costos directos generados

\section{Intervención}

De los 336 niños enrolados, 168 fueron aleatorizados a recibir la intervención y 168 , al grupo control. Recibieron el suplemento de LBR a dosis de 1x108 UFC (cinco gotas durante la primera comida del día) o placebo durante 12 semanas.

Para prevenir la deshidratación fueron prescriptas las sales de rehidratación oral de la Organización Mundial de la Salud; ante la fiebre fue recomendado el uso de antipiréticos y ante un cuadro febril de más de 72 horas de evolución, fue prescripto tratamiento antibiótico con amoxicilina-clavulánico durante siete días.

El análisis de costo-efectividad fue realizado desde la perspectiva del tercer pagador incluyendo solamente los costos directos de cada una de ambas alternativas

\section{Resultados}

No hubo diferencias en las características basales de ambos grupos ni pérdidas de participantes durante el estudio. Los principales resultados se describen en la tabla 1.

Tabla 1: eficacia de la suplementación con Lactobacillus reuteri en niños sobre la incidencia y la duración de los episodios de diarrea y de infecciones respiratorias.

\begin{tabular}{|c|c|c|c|c|}
\hline \multicolumn{2}{|l|}{ Resultado } & $\begin{array}{c}\text { L. reuteri } \\
n=168\end{array}$ & $\begin{array}{c}\text { Placebo } \\
n=168\end{array}$ & $\mathbf{p}$ \\
\hline \multirow[t]{2}{*}{ Cantidad total de episodios de diarrea } & Durante la intervención & 42 & 69 & 0,03 \\
\hline & Durante el seguimiento & 57 & 83 & 0,04 \\
\hline \multirow[t]{2}{*}{ Duración promedio de los episodios de diarrea } & Durante la intervención & 1,4 & 2,5 & 0,01 \\
\hline & Durante el seguimiento & 1,6 & 2,4 & 0,01 \\
\hline \multirow{2}{*}{$\begin{array}{l}\text { Cantidad promedio de días con síntomas de } \\
\text { infecciòn respiratoria }\end{array}$} & Durante la intervención & 1,5 & 4,6 & 0,01 \\
\hline & Durante el seguimiento & 2,1 & 4,4 & 0,01 \\
\hline
\end{tabular}

En ninguno de los dos grupos fueron documentados efectos adversos severos, asociándose el uso de L. reuteri a una reducción promedio de 36 dólares por cada caso de diarrea y de 37 dólares por cada caso de infección de tracto respiratorio evitados (estrategias dominantes).

\section{Conclusión}

La suplementación diaria con L. reuteri en niños sanos que concurren a guarderías se asoció con una reducción significativa del número de episodios de diarrea y de su duración, y del número de infecciones del tracto respiratorio. La estrategia mostró ser "costo-ahorrativa".

Fuente de financiamiento: BioGaia AB, Estocolmo, Suecia.

\section{Comentario}

Las enfermedades diarreicas son la segunda causa de muerte en menores de cinco años y una de las principales causas de desnutrición en ese rango etario. A nivel mundial ocurren aproximadamente 1,5 mil millones de episodios de diarrea por año, que conducen a la muerte a casi dos millones de menores de cinco años ${ }^{1}$.

Consideramos que la metodología de esta investigación ha sido prolija, con una población bien definida a través de criterios de inclusión y exclusión claros, grupos bien balanceados en lo que respecta a potenciales factores de confusión, y

escasa pérdida de participantes en el seguimiento, lo que permite garantizar una buena validez interna.

\section{Conclusiones de la comentadora}

Si bien sus hallazgos de esta investigación fueron estadísticamente significativos, nos quedan dudas respecto de su relevancia clínica, ya que la suplementación con L. reuteri no logró reducir más de 24 horas la duración de los episodios diarreicos (beneficio atribuible a la intervención) por lo que demuestra que el producto no modificaría significativamente el cuadro.

Fuente de financiamiento: beca de BioGaia $A B$, Suecia. Conflicto de intereses: no presenta.

Cintia Martinez [ Servicio de Medicina Familiar y Comunitaria del Hospital Italiano de Buenos Aires. ayelen.martinez@ hiba.org.ar]

Martínez C. La suplementación con Lactobacillus reuteri fue eficaz y costo ahorrativa para prevenir diarreas e infecciones respiratorias en preescolares. Evid Act Pract Ambul. 2015;18(4):113. Oct-Dic. Comentado de: Gutierrez-Castrellon P y col. Diarrhea in Preschool Children and Lactobacillus reuteri: A Randomized Controlled Trial. Pediatrics 2014; 133 (4): 904-9.

\section{Referencias:}

1. Farthing M y col. Guía Práctica de la Organización Mundial de Gastroenterología Diarrea aguda en adultos y niñosuna perspectiva mundial. Organización Mundial de Gastroenterología, 2012. Disponible en URL: http://www.worldgastroenterology.org/UserFiles/file/guidelines/acute-diarnea-spanish-2012.pdf (último acceso 24/11/15). 